SHORT REPORT

\title{
Securing central venous catheters: a comparison of sutures with staples
}

\author{
A Vinjirayer, P Jefferson, D R Ball
}

Emerg Med J 2004;21:582-583. doi: 10.1136/emj.2003.009860

Objectives: Central venous access permits rapid drug delivery to the central circulation during cardiopulmonary resuscitation. Central venous catheters must be secured in place to prevent accidental removal and sutures are often used for this purpose. Staples may offer advantages over sutures by reducing the time needed to secure the central venous catheter and reducing exposure to contaminated sharps. The purpose of this study was to assess a staple anchoring device (Arrow, Reading, USA) and compare it with sutures for securing central venous catheters.

Methods: Prospective, randomised trial of 20 patients requiring insertion of a central venous catheter during elective surgery.

Results: Mean (SD) times were significantly shorter in the staples group than in the sutures group (staples 10.1 (3.5) seconds; sutures 50.5 (9.6) seconds; $p<0.0001$ ). However, 3 of 10 central venous catheters in the staples group were accidentally pulled out within the first three days. Conclusions: The use of staples significantly reduces the time to secure a central venous catheter. However, staples failed to secure the central venous catheters adequately and therefore cannot be recommended instead of sutures.

C entral venous catheters (CVC) are routinely used in many medical specialties. During cardiopulmonary resuscitation central, venous access permits rapid drug delivery to the central circulation when compared with peripheral venous access. Catheters must be secured in place to prevent inadvertent removal and sutures are often used for this purpose.

The staple anchoring device (Arrow, Reading, USA, fig 1) is a commercially available alternative to sutures. This consists of a disposable staple applicator containing a single staple.

The potential benefits from using staples instead of sutures include a reduction in the time spent securing the CVC in place and a reduction in the risk of needlestick injury. Staples are already used in emergency departments and are a fast and cost effective alternative to sutures for the repair of scalp lacerations.

The aim of this study was to assess the staple anchoring device and compare the time needed to secure a CVC using sutures or staples.

\section{METHODS}

The study was approved by the local research ethics committee and written, informed consent was obtained from 20 patients undergoing major elective surgery in whom the anaesthetist planned to insert a CVC.

Sealed envelopes were used to randomise the patients to have their CVC secured with staples or sutures.
All CVC were inserted into the right internal jugular vein after the induction of general anaesthesia. After insertion of the CVC, the anaesthetist was provided with either a suture or two staple applicators that were placed on a sterile tray. The time to secure the catheter was measured from when the needle or first staple applicator was picked up until the needle or second empty staple applicator was returned to the tray. The time was measured to the nearest second.

Arrow 8.5 F quad-lumen CVC (Arrow, Reading, USA.) were used and were secured at the catheter clamp but not at the catheter hub (fig 2), which is the usual practice of one of the authors. One suture or one staple was used in each eye of the hub. Hand held curved sutures were used. The catheters were covered with a transparent dressing.

Ease of use of the fixation device (staple or suture) was rated as easy, moderate, or difficult.

Any needlestick injuries or other problems related to securing the CVC were noted.

Patients were reviewed every day for three days postoperatively or until the CVC was removed if earlier and any adverse events were recorded. An adverse event was defined as unintentional removal of the CVC or failure of the securing device. Any other relevant problems were also recorded.

The times taken to secure a CVC were compared using the Mann-Whitney U test. A p value $<0.05$ was regarded as significant. We assumed that a $50 \%$ reduction in time would be clinically significant. Power analysis indicated that a minimum of six patients were required to detect this difference with a power of $90 \%$.

\section{RESULTS}

No difficulties were encountered using the staples or sutures and all fixations were graded as easy. No needlestick injuries were recorded (table 1 ).

Three adverse events were recorded in the staples group. All were related to accidental removal of the CVC during

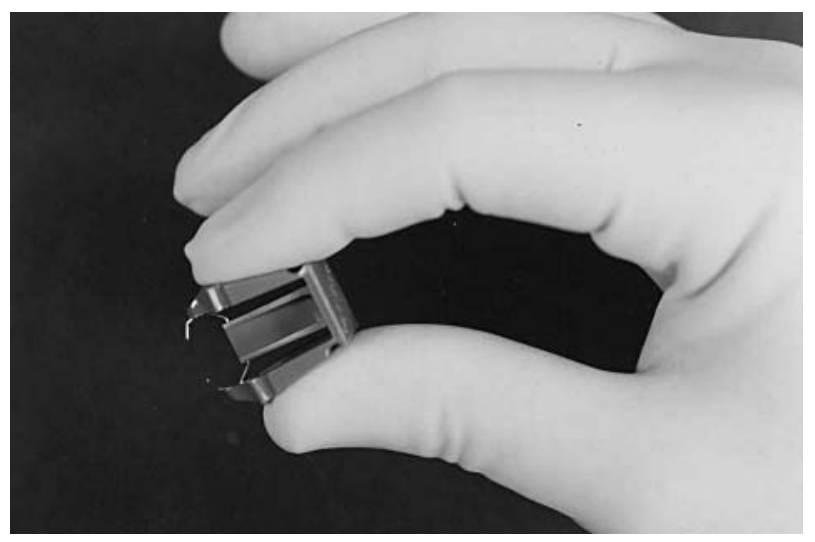

Figure 1 Staple anchoring device. 


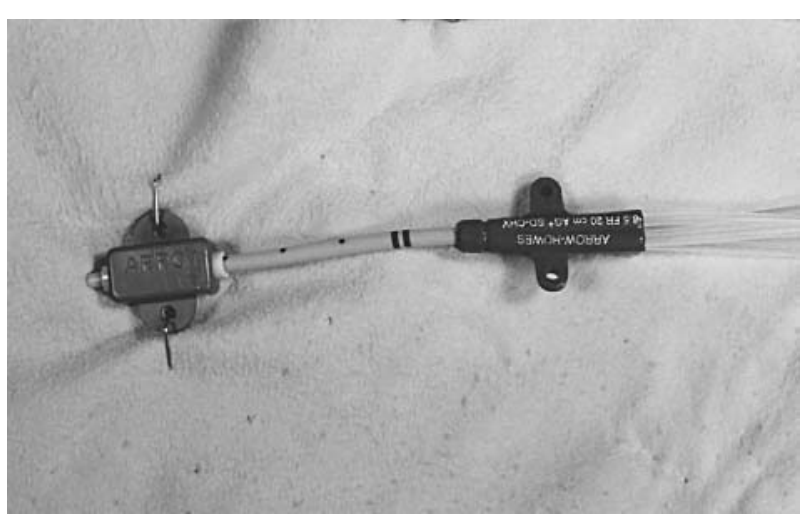

Figure 2 CVC secured at catheter clamp.

patient transfers. On two occasions, the CVC was pulled out through the catheter clamp, but the clamp and staples remained in place. On the other occasion, the CVC, clamp and staples were pulled out together.

\section{DISCUSSION}

Our study shows that a CVC can be secured much more quickly using the staple anchoring device instead of sutures. A reduction in mean time of 40 seconds may not be of importance in an elective setting but may be more relevant in an emergency situation.

Another benefit of staples is the potential to reduce needlestick injury. Needlestick injury is not uncommon in medical staff, with 39\% of anaesthetists reporting such an injury in a 12 month period. ${ }^{2}$ Guidelines regarding precautions against infection are available ${ }^{3}$ but it has been well reported that medical staff do not always follow recommendations about sharps disposal or other precautions. In 1999, over $45 \%$ of anaesthetists rarely or never wore gloves ${ }^{4}$ and $73 \%$ re-sheathed needles during routine practice. ${ }^{5}$ Reducing exposure to contaminated sharps may be a way of reducing the risk of needlestick injury during CVC insertion. Staples remove the need for a suture needle and thus decrease the number of contaminated sharps. Once again, this advantage may be more apparent in a resuscitation or emergency environment where sharp disposal may not be seen as a priority, and the patient population has a high incidence of illegal drug use. ${ }^{6}$

However, the potential benefits of staples do not outweigh its poor performance as a securing device. Three CVC secured with staples were inadvertently pulled out within three days. This could have serious consequences if a patient is receiving inotropic support and is potentially fatal.
Table 1 Time to secure CVC and adverse events. Results are expressed as mean (SD) or number

\begin{tabular}{lll}
\hline & Sutures $(\mathbf{n}=10)$ & Staples $(\mathbf{n}=10)$ \\
\hline Time $(\mathrm{s})$ & $50.5(9.6)$ & $10.1(3.5)^{*}$ \\
Needlesticks & 0 & 0 \\
Adverse events & 0 & 3 \\
\hline${ }^{*} \mathrm{p}<0.0001$. &
\end{tabular}

It is possible that the performance of the staples would have been improved if the CVC were secured at both the catheter clamp and the catheter hub. We decided to secure the CVC only at the catheter clamp as this was the routine practice of one of the authors.

Our results suggest that the staple anchoring device does not adequately secure a CVC and that the advantage of time saved in securing the CVC does not outweigh the consequences of accidental CVC removal.

\section{ACKNOWLEDGEMENTS}

We would like to thank the Research and Development Department at Dumfries and Galloway Royal Infirmary, and Arrow for supplying the staple anchoring devices.

\section{Authors' affiliations}

A Vinjirayer, P Jefferson, D R Ball, Department of Anaesthetics and Intensive Care, Dumfries and Galloway Royal Infirmary, Dumfries, UK

Funding: none.

Conflicts of interest: none declared.

Correspondence to: Dr P Jefferson, Department of Anaesthetics and Intensive Care, Dumfries and Galloway Royal Infirmary, Bankend Road, Dumfries DG1 4AP, UK; p.jefferson@dgri.scot.nhs.uk

Accepted for publication 13 August 2003

\section{REFERENCES}

1 Hogg K, Carley S. Towards evidence based emergency medicine: best BETs from the Manchester Royal Infirmary. Staples or sutures for repair of scalp lacerations in adults. Emerg Med J 2002;19:327-8.

2 Richards MJ, Jenkin GA, Johnson PD. Universal precautions: attitudes of Australian and New Zealand anaesthetists. Med J Aust 1997;166:138-40.

3 Association of Anaesthetists of Great Britain and Ireland. HIV and other blood borne viruses-guidance for anaesthetists. London: Association of Anaesthetists of Great Britain and Ireland, 1992.

4 El Mikatti N, Dillon P, Healey TEJ. Hygienic practices of consultant anaesthetists: a survey in the North-West region of the UK. Anaesthesia 1999;54:13-18.

5 Wise HJ, McCormick RN. Re-enforcing hygiene practices of anaesthetists. Anaesthesia 1999;54:1220-1.

6 Carriga TD, Field $\mathrm{H}$, Illingworth RN, et al. Toxicological screening in trauma. J Accid Emerg Med 2000;17:33-7. 